\title{
Multimorbidity and Mental Health-Related Quality of Life and Risk of Completed Suicide
}

\author{
Melissa Y. Wei, MD, MPH, MS* and Kenneth J. Mukamal, MD, MPH, MA ${ }^{\dagger}$
}

BACKGROUND: Physical functioning indexed multimorbidity is strongly associated with long-term mortality, but its role in poor mental health has not been quantified.

METHODS: A total of 252002 community-dwelling adults in the Nurses' Health Study (NHS), NHS II, and Health Professionals Follow-up Study (HPFS) prospective cohorts reported physician-diagnosed diseases and the Short Form-36 over 8 years and had 24-year follow-up for suicide mortality. We quantified multimorbidity using a multimorbidity-weighted index (MWI). We used multivariable-adjusted proportional hazards models with competing risks for suicide mortality and mixed-effects models to estimate mental health-related quality of life (HRQOL).

RESULTS: Multimorbidity was associated with an increased risk of suicide mortality in an approximately linear manner, with roughly two- to threefold higher risk in adults with the highest vs lowest quartile MWI in adjusted models: NHS hazard ratio $(\mathrm{HR})=3.01 \quad(95 \%$ confidence interval $[\mathrm{CI}]=1.48-6.11)$; NHS II HR = 3.04 (95\% CI = 1.82-5.09); HPFS HR $=1.74(95 \% \mathrm{CI}=1.08-2.81)$. Greater MWI was associated with worse mental HRQOL 8 years later across all scales and the mental component summary (MCS) in a dose-response manner. This association was attenuated but persisted after adjustment for baseline mental HRQOL and other covariates. Adults with the highest quartile MWI had lower MCS in adjusted models compared with those with

From the *Division of General Medicine and Institute for Healthcare Policy and Innovation, University of Michigan, Ann Arbor, Michigan; and the ${ }^{\dagger}$ Division of General Medicine and Primary Care, Beth Israel Deaconess Medical Center and Harvard Medical School, Brookline, Massachusetts.

Address correspondence to Melissa Wei, MD, MPH, MS, Division of General Medicine, University of Michigan Medical School, 2800 Plymouth Road, Building 16, Room 430W, Ann Arbor, MI 48109;

E-mail: weimy@med.umich.edu

Funding Information. This study was supported by the National Institutes of Health (grants UM1 CA186107 for the Nurses' Health Study, UM1 CA176726 for the Nurses' Health Study II, and UM1 CA167552 for the Health Professionals Follow-up Study). Melissa Wei was supported through a Career Development Award from the National Institutes of Health, National Institute on Aging (K23 AG056638).

DOI: 10.1111 /jgs. 15678 the lowest quartile MWI: NHS $\beta=-0.61(95 \% \mathrm{CI}=-0.78$ to -0.44$)$; NHS II $\beta=-1.25$ (95\% CI $=-1.44$ to -1.06$)$.

CONCLUSION: Multimorbidity is associated with substantially higher suicide mortality risk and worse mental HRQOL across all available scales, even when indexed to physical functioning. These results highlight the substantial mental health burden imposed by multimorbidity at all ages and sexes. J Am Geriatr Soc 67:511-519, 2019.

Key words: Multimorbidity; mental health; healthrelated quality of life; Short Form-36; suicide mortality

S uicide consistently ranks among the top 10 causes of death for US adults. ${ }^{1}$ Between 1999 and 2016, suicide rates increased $28 \%$ for men and women ages 10 to 74 years. $^{2}$ Within 4 weeks of death, half used a healthcare service (outpatient, inpatient, emergency department visits), and in the year prior, $83 \%$ made a healthcare visit. ${ }^{3}$ Despite this, providers lack effective tools for screening and intervening beyond traditional risk factors such as mental health ${ }^{4}$ and substance use conditions ${ }^{5}$ or suicidal ideation.

Older adults have a high burden of physical illness that adversely impacts mental health and vice versa. ${ }^{6}$ Physical conditions that are incurable and/or severe, such as acquired immunodeficiency syndrome, are independent risk factors for suicidal ideation ${ }^{7,8}$ and mortality. 9 Although prior studies established mental and physical conditions as risk factors for suicide in older adults, quantifying this relationship has generally taken a disease-specific approach and relied on case-control designs. ${ }^{9-11}$ However, diseases are dynamic and rarely remain in isolation over the life span. ${ }^{12}$ Multimorbidity, the coexistence of multiple chronic conditions and most common experience among patients, has increased over time and is particularly prevalent in older adults, ranging from $26 \%$ in noninstitutionalized adults to $71 \%$ in hospitalized adults between 2009 and 2012. ${ }^{13}$ Suicide risk due to multimorbidity remains poorly understood.

Multimorbidity may be a useful construct to quantify cumulative burden of chronic mental and physical illness and 
help clinicians screen for suicide risk, but comprehensive tools to measure it have been limited. We developed and validated a patient-centered measure of multimorbidity, the multimorbidityweighted index (MWI), ${ }^{14,15}$ that weights diseases to patientreported physical functioning. MWI is strongly associated with physical and cognitive function and mortality and captures a broader range of multimorbidity than other indices. ${ }^{14,15}$ Importantly, the Short Form (SF)-36 physical functioning scale to which MWI is weighted is completely uncorrelated with SF36's mental health-related quality of life (HRQOL) scales, by design. ${ }^{16}$ Thus MWI represents a unique instrument with which to gauge the full burden of multimorbidity on mental health, for its weighting would minimize the role of multimorbidity on mental HRQOL, and it accurately quantifies both low and high degrees of multimorbidity.

To assess prospective associations of multimorbidity, as estimated by MWI, with mental HRQOL and risk of suicide mortality, we studied three large cohorts of young through older community-dwelling adults with over 24-year followup and, in two cohorts, repeated assessments of mental HRQOL. We sought to quantify the longitudinal burden imposed by the full range of multimorbidity on two related outcomes.

\section{METHODS}

\section{Study Population}

We included three nationally-sampled prospective cohorts of US community-dwelling participants, the Nurses' Health Study (NHS), NHS II, and Health Professionals Follow-up Study (HPFS). NHS was established in 1976 when 121701 female nurses aged 30 to 55 years completed a mailed questionnaire on diet, lifestyle, and medical history. A younger cohort of female nurses, NHS II, was established in 1989 and enrolled 116686 nurses aged 25 to 42 years who responded to a similar questionnaire. HPFS enrolled 51530 male health professionals aged 40 to 75 years in 1986 who also submitted biennial questionnaires. Participants reported physician-diagnosed chronic conditions in 1992-1993 and had follow-up on HRQOL through 2000-2001 and suicide mortality through 2016. We excluded pregnant women because pregnancy is associated with reduced HRQOL. ${ }^{17}$ This study was approved by a blinded institutional review board.

\section{Multimorbidity Measurement}

Multimorbidity was assessed using the validated MWI. ${ }^{14,15}$ Briefly, chronic conditions were weighted by their estimated impact on physical functioning and then summed to form each individual's MWI. MWI represents an individual's cumulative burden of chronic conditions. Further, each 1-point increase in MWI conveniently calibrates to a 1 -point decrease in SF-36 physical functioning. For this analysis, we applied our previously established weights to 65 available conditions reported in 1992-1993 to create a baseline MWI for each participant.

Depression and substance abuse disorders were not assessed as self-reported conditions at baseline so were not included in the MWI in this study. For robustness, we included these conditions as defined by regular antidepressant and tranquilizer use, available in NHS II and HPFS. We assigned the MWI weighting for depression to antidepressant use, and used the weighting for alcohol abuse to impute a weighting for tranquilizer use. Alcohol abuse was assessed in NHS II only.

\section{Suicide Mortality Assessment}

Deaths through December 31, 2016, were identified at the end of each 2-year cycle through the National Death Index. ${ }^{18,19}$ Suicide was defined using International Classification of Diseases, Revision 8, codes for suicide and selfinflicted injury (E950-E959). Cases included suicide and self-inflicted poisoning by solid or liquid substances, gases in domestic use, or other gases; suicide and self-inflicted injury by hanging, strangulation and suffocation, submersion, firearms and explosives, cutting and piercing instruments, jumping from high place, other and unspecified means; and late effects of self-inflicted injury.

\section{HRQOL Assessment}

SF-36 is among the most widely used health survey instruments to assess HRQOL and was extensively validated. ${ }^{20}$ It includes eight scales and two summary component scores self-reported in 1992 and 2000 (NHS) and 1993 and 2001 (NHS II). In HPFS, only physical functioning was assessed, and its association with MWI was reported previously. ${ }^{21}$

SF-36 comprises eight overlapping scales: physical functioning, role limitations due to physical health problems (role-physical), bodily pain, general health, vitality, social functioning, role limitations to emotional health problems (role-emotional), and mental health. The mental component summary (MCS) and physical component summary (PCS) are computed based on the eight scales and are orthogonal (uncorrelated) by design, ${ }^{16}$ precluding risk factors that influence physical HRQOL from affecting mental HRQOL. MCS includes social functioning, role-emotional, mental health, general health, and vitality. PCS includes physical functioning, role-physical, bodily pain, general health, and vitality. General health and vitality are in both MCS and PCS. We used MCS as our primary mental HRQOL outcome and examined individual mental health subscales for robustness. We also present results for physical HRQOL as a comparator; we previously reported strong effects of MWI on physical HRQOL, as expected from its weighting.

Each of the scales and summary component scores range from 0 to 100 , with higher scores indicating better HRQOL. Scores are calibrated so the mean for the general US population on each scale, and summary is 50 with a standard deviation of $10 .^{16}$

\section{Covariate Assessment}

We selected covariates considered potentially important predictors for multimorbidity and HRQOL. ${ }^{22-27}$ These included age, race, body mass index, smoking status, geographic region, alcohol intake, coffee intake, marital status, and living alone. Additionally, in NHS, religious service attendance was available and adjusted for due to its strong inverse association with suicide mortality. ${ }^{27}$ Participant characteristics were 
assessed at baseline in 1992 except alcohol and coffee intake, assessed in 1990.

\section{Statistical Analysis}

We conducted analyses for two sets of outcomes, suicide mortality and mental HRQOL. For the first set of analyses, suicide mortality, we used multivariable Cox proportional hazards regression models with censoring for nonsuicide mortality and nondeaths ${ }^{28}$ to determine the association of MWI with suicide mortality. We calculated hazard ratios (HRs) and $95 \%$ confidence intervals (CIs) comparing each 1-point increase in MWI and quartiles of MWI through Cox proportional hazards modeling and the Efron $^{29}$ method for handling ties. We assessed cohorts separately; then, given similar trends, we pooled results using fixed-effects meta-analysis to obtain the DerSimonianLaird estimator. $^{30}$

To assess for a potential nonlinear association between MWI and suicide mortality, we used restricted cubic splines $^{31}$ and winsorized the top 5\% MWI values to reduce the possible effect of spurious outliers. ${ }^{32}$ Nonlinearity was tested through the likelihood ratio test to compare models with one linear term vs both linear and cubic spline terms. We presented Kaplan-Meier survival curves for suicide mortality by MWI quartiles.

For our second outcome, mental HRQOL, we used mixed-effects models to examine MWI and all SF-36 scales and summary component scores. MWI was assessed continuously and categorically with each of eight SF-36 scales and two summary measures.

For both sets of analyses, we adjusted for all covariates. Given the strong correlation between current and future HRQOL, we adjusted for each respective SF-36 scale or summary measure at baseline to minimize the potential influence of current HRQOL. Despite potential overadjustment, the regression coefficient provides a prediction and is preferred over change scores. ${ }^{33}$ Analyses used Statistical Analysis System software v.9.4 (SAS Institute, Cary, NC, 2013).

\section{RESULTS}

\section{Participant Characteristics}

We included 252002 participants $(\mathrm{N}=102854$ NHS, $\mathrm{N}=103584$ NHS II, $\mathrm{N}=45564$ HPFS) who completed the biennial questionnaire in 1992 or 1993 and had complete data on physician-diagnosed chronic conditions to compute MWI. Table 1 shows the baseline characteristics stratified by median MWI. On average, MWI was higher in older women (NHS) than men and younger women (NHS II).

At baseline, mean SF-36 scales and summary component measures were close to general population norms (Supplemental Table S1). On average, younger women had lower MCS and higher PCS than older women, consistent with US normative data, whereby mental HRQOL improves with age while physical HRQOL declines. ${ }^{16}$

After follow-up through 2000-2001, the mean age was age 66.0 (7.1) in NHS and 46.5 (4.6) years in NHS II. The SF-36 physical functioning scale that was used to weight diseases in MWI was moderately correlated with PCS after follow-up (Pearson's $r=0.56$ in NHS, $r=0.45$ in NHS II). Physical functioning was only weakly correlated with MCS at follow-up (Pearson's $r=0.06$ in NHS, $r=0.09$ in NHS II), as expected due to the orthogonal design of SF-36 physical and mental health constructs. MCS and all mental HRQOL scales increased while PCS and all physical HRQOL scales declined (Supplemental Table S1).

\section{Multimorbidity and Risk of Suicide Mortality}

There were 341 cases of suicide mortality between 1992-1993 and 2016. The greatest absolute number and rate of suicide occurred in men $(\mathrm{N}=154,14.1 / 100000)$, compared with younger women in NHS II $(\mathrm{N}=110$, $4.62 / 100000)$ and older women in NHS $(\mathrm{N}=77,3.12 / 100$ 000). For causes of death, suicide comprised the highest percentage in younger women in NHS II (3.8\% deaths) compared with men $(0.79 \%$ deaths $)$ and older women in NHS $(0.22 \%$ deaths). The most common suicide method in women was poisoning by solid or liquid substances; in men, it was firearms and explosives.

We assessed for nonlinearity using fractional polynomials and restricted cubic splines. Based on restricted cubic splines (but not fractional polynomials), nonlinearity occurred at high MWI values, but we observed essentially linear relationships with mortality through MWI values of 12 to 15 for NHS, 14 to 18 for HPFS, and 20 to 26 for NHS II (Figure 1). Although extreme MWI values increased mortality disproportionately, MWI had an essentially linear relationship with suicide mortality throughout values commonly observed in community-dwelling adults.

In unadjusted models, each point increase in MWI was significantly associated with $9 \%$ to $19 \%$ higher suicide mortality in the three cohorts (Table 2; Supplemental Table S2). In fully adjusted models, there was mild attenuation to $5 \%$ to $15 \%$ higher suicide mortality for each point increase in MWI. These results were significant for women and men.

The association between multimorbidity and suicide mortality was stronger for women than men. In fully adjusted models, suicide mortality risk tripled for younger and older women in the highest vs lowest quartile MWI (Table 2). After including regular use of antidepressants and tranquilizers as morbidities in MWI, the risk of suicide mortality was even greater. NHS II women with the highest quartile MWI had more than a 4.5 -fold increased risk of suicide mortality vs those in the lowest quartile $(\mathrm{HR}=4.64,95 \% \mathrm{CI}=2.68-8.03)$ in fully adjusted models (Supplemental Table S2).

In men, suicide mortality risk was nearly double for those in the highest vs lowest quartile MWI ( $\mathrm{HR}=1.74,95 \%$ $\mathrm{CI}=1.08-2.81)$. After including morbidities from antidepressant and tranquilizer use, suicide mortality risk doubled $(\mathrm{HR}=2.01,95 \% \mathrm{CI}=1.23-3.28)$ in fully adjusted models.

In models of MWI that included medication use, there was suggestion of a dose-response association with greater MWI and increased suicide mortality. Kaplan-Meier survival curves for suicide mortality for all cohorts using the original MWI based on self-reported conditions only are shown in Figure 2.

For individuals with physical conditions only (models excluded those with mental health and substance use conditions), increased suicide mortality risk persisted (Supplemental Table S3). 
Table 1. Multimorbidity Characteristics of Participants ${ }^{\mathrm{a}}$

\begin{tabular}{|c|c|c|c|c|c|c|}
\hline \multirow[b]{2}{*}{ MWI median cut point } & \multicolumn{2}{|c|}{ NHS } & \multicolumn{2}{|c|}{ NHS II } & \multicolumn{2}{|c|}{ HPFS } \\
\hline & $\begin{array}{l}\text { MWI }<\text { median } \\
\quad(0-1.86)\end{array}$ & $\begin{array}{l}\mathrm{MWI} \geq \text { median } \\
(1.87-38.2)\end{array}$ & $\begin{array}{l}\text { MWI < median } \\
\quad(0-0.33)\end{array}$ & $\begin{array}{c}\mathrm{MWI} \geq \text { median } \\
(0.34-27.1)\end{array}$ & $\begin{array}{l}\text { MWI < median } \\
\quad(0-1.18)\end{array}$ & $\begin{array}{l}\mathrm{MWI} \geq \text { median } \\
(1.19-29.9)\end{array}$ \\
\hline No. of participants (\%) & $49573(48.2)$ & 53358 (51.8) & 47493 (45.9) & 56091 (54.1) & $22813(50.1)$ & 22751 (49.9) \\
\hline Age, y, mean (SD) & $56.4(7.0)$ & $60.2(6.9)$ & $37.7(4.6)$ & $39.0(4.5)$ & $57.1(8.8)$ & $63.7(9.5)$ \\
\hline White race $(\%)$ & 46565 (93.9) & 49812 (93.3) & 44300 (93.3) & 52292 (93.2) & 20633 (90.4) & 20496 (90.1) \\
\hline $\begin{array}{l}\text { Body mass index, } \mathrm{kg} / \mathrm{m}^{2} \text {, } \\
\text { mean (SD) }\end{array}$ & $25.7(4.7)$ & $27.1(5.6)$ & $24.2(4.7)$ & $26.3(6.1)$ & 25.5 & $26.1(3.6)$ \\
\hline$<18.5(\%)$ & $685(1.4)$ & 727 (2.2) & $1203(2.5)$ & $1064(1.9)$ & $66(0.29)$ & $69(0.30)$ \\
\hline $18.5-24.9(\%)$ & 26226 (52.9) & 21022 (39.4) & $31331(66.0)$ & 28727 (51.2) & $10451(45.8)$ & $9167(40.3)$ \\
\hline $25-29.9(\%)$ & 15506 (31.3) & 18293 (34.3) & $9817(20.7)$ & $13832(24.7)$ & $10454(44.8)$ & $10786(47.4)$ \\
\hline $30-34.9(\%)$ & $5166(10.4)$ & $8614(16.1)$ & $3354(7.1)$ & $6929(12.4)$ & $1588(7.0)$ & $2213(9.7)$ \\
\hline$\geq 35(\%)$ & $1990(4.0)$ & $4702(8.8)$ & $1788(3.8)$ & $5539(9.9)$ & $254(1.1)$ & $516(2.3)$ \\
\hline \multicolumn{7}{|l|}{ Tobacco abuse } \\
\hline Never (\%) & $22390(45.2)$ & 22654 (42.5) & 32139 (67.7) & 35010 (62.4) & 10849 (47.6) & $8637(38.0)$ \\
\hline Former (\%) & 19010 (38.4) & 23023 (43.1) & 10422 (21.9) & 13835 (24.7) & 8746 (38.3) & 11007 (48.4) \\
\hline Current (\%) & $8173(16.5)$ & $7681(14.4)$ & $4932(10.4)$ & 7246 (12.9) & $1652(7.2)$ & $1601(7.0)$ \\
\hline \multicolumn{7}{|l|}{ Geographic region } \\
\hline West (\%) & 6007 (12.1) & $6670(12.5)$ & $6036(12.7)$ & 7354 (13.1) & 5448 (23.9) & 4957 (21.8) \\
\hline Midwest (\%) & $8921(18.0)$ & 10423 (19.5) & $13152(27.7)$ & 15926 (28.4) & $6225(27.3)$ & $6193(27.2)$ \\
\hline Northeast (\%) & 31023 (62.6) & 32031 (60.0) & 13835 (29.1) & 16760 (29.9) & $6282(27.5)$ & $6556(28.8)$ \\
\hline South (\%) & $3622(7.3)$ & $4234(7.9)$ & 8276 (17.4) & 7936 (14.2) & $4831(21.2)$ & $5033(22.1)$ \\
\hline Married (\%) & 33958 (83.0) & $37970(80.0)$ & 33517 (70.6) & 38535 (68.7) & 17755 (77.8) & 17961 (79.0) \\
\hline Lives alone (\%) & 4115 (8.3) & $6162(11.6)$ & 44007 (92.7) & $4878(8.7)$ & 1407 (6.2) & $1395(6.1)$ \\
\hline $\begin{array}{l}\text { Religious service } \\
\text { attendance }\end{array}$ & & & NA & NA & NA & NA \\
\hline Never (\%) & 8239 (23.9) & 9572 (23.8) & & & & \\
\hline$<1 /$ wk $(\%)$ & $7803(22.6)$ & 9059 (22.5) & & & & \\
\hline$\geq 1 /$ wk (\%) & 18443 (53.4) & $21562(53.6)$ & & & & \\
\hline $\begin{array}{l}\text { Alcohol intake, } \mathrm{g} / \mathrm{d} \text {, mean } \\
\text { (SD) }\end{array}$ & $5.3(9.5)$ & $4.8(9.5)$ & $3.1(6.1)$ & $3.0(6.1)$ & $9.9(13.5)$ & $10.5(15.0)$ \\
\hline Medication use $\mathrm{b}^{\mathrm{b}}$ & NA & NA & & & & \\
\hline Antidepresssant & & & $2597(5.5)$ & 8918 (15.9) & $598(1.3)$ & $591(1.3)$ \\
\hline Mild tranquilizer (\%) & & & $302(0.6)$ & $1475(2.6)$ & 890 (1.9) & $882(1.9)$ \\
\hline Major tranquilizer (\%) & & & $35(0.1)$ & $146(0.3)$ & $33(0.1)$ & $33(0.1)$ \\
\hline \multicolumn{7}{|l|}{ Coffee intake, cups/d } \\
\hline $0(\%)$ & $9591(26.8)$ & 12837 (31.0) & 15399 (32.4) & $18370(32.7)$ & $5622(24.6)$ & 6047 (26.6) \\
\hline$<1(\%)$ & 4526 (12.7) & 5629 (13.6) & $6610(13.9)$ & 7635 (13.6) & 3517 (15.4) & $3810(16.8)$ \\
\hline $1(\%)$ & $5123(14.3)$ & $6526(15.8)$ & $5278(11.1)$ & $6196(11.1)$ & 2586 (11.3) & 2767 (12.2) \\
\hline $2-3(\%)$ & 11861 (33.2) & $12021(29.1)$ & $10303(21.7)$ & 11966 (21.3) & $4558(20.0)$ & 4194 (18.4) \\
\hline$\geq 4(\%)$ & $4868(13.6)$ & $4353(10.5)$ & $3563(7.5)$ & $4828(8.6)$ & $1924(8.44)$ & $1524(6.7)$ \\
\hline $\begin{array}{l}\text { Baseline Short Form-36, } \\
\text { mean (SD) }\end{array}$ & & & & & NA & NA \\
\hline $\begin{array}{l}\text { Physical Component } \\
\text { Summary (PCS) }\end{array}$ & $53.2(6.6)$ & $45.0(10.3)$ & $55.4(5.7)$ & $51.0(8.8)$ & & \\
\hline $\begin{array}{l}\text { Mental Component } \\
\text { Summary (MCS) }\end{array}$ & $51.8(8.2)$ & $51.8(9.2)$ & $48.6(8.8)$ & $46.7(10.1)$ & & \\
\hline $\begin{array}{l}\text { Multimorbidity-Weighted } \\
\text { Index, mean (SD) }\end{array}$ & $0.44(0.59)$ & $5.6(3.5)$ & $0.005(0.03)$ & $2.51(2.4)$ & $0.26(0.41)$ & $4.24(3.0)$ \\
\hline $\begin{array}{l}\text { Disease count, mean } \\
\text { (SD) }\end{array}$ & $0.63(0.76)$ & $3.2(1.8)$ & $0.02(0.14)$ & $1.86(1.2)$ & $0.59(0.76)$ & $3.42(2.0)$ \\
\hline
\end{tabular}

${ }^{\mathrm{a} B}$ By MWI median at baseline in 1992 (NHS I, HPFS) or 1993 (NHS II).

${ }^{\mathrm{b}}$ Current regular medication use of antidepressants (tricyclic antidepressants, fluoxetine, etc), minor tranquilizers (eg, diazepam, alprazolam, lorazepam, clonazepam, chlordiazepoxide, etc), and major tranquilizers (eg, haloperidol, trifluoperazine, chlorpromazine, fluphenazine, thioridazine, perphenazine, etc). HPFS = Health Professionals Follow-up Study; MCS = mental component summary; MWI = multimorbidity-weighted index; NA = not available; NHS = Nurses' Health Study; NHS II = Nurses' Health Study II; PCS = physical component summary; SD = standard deviation.

\section{Multimorbidity and Mental HRQOL}

Associations between multimorbidity and mental HRQOL 8 years later are shown (Supplemental Tables S4 and S5 and Figure 3). Older women in NHS with the highest quartile MWI had a lower MCS than those with the lowest quartile MWI $(\beta=-0.83,95 \% \mathrm{CI}=-0.99$ to $-0.68)$. After adjustment for baseline MCS and covariates, the association was attenuated to a $-0.61(95 \%$ $\mathrm{CI}=-0.78$ to -0.44$)$ point worse MCS. A similar pattern of attenuation in MCS after adjustment was 
A NHS 1992-2016 mortality by suicide

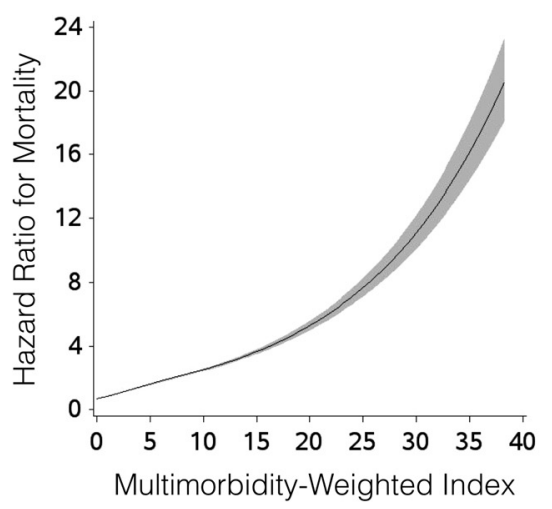

B NHS II 1993-2016 mortality by suicide

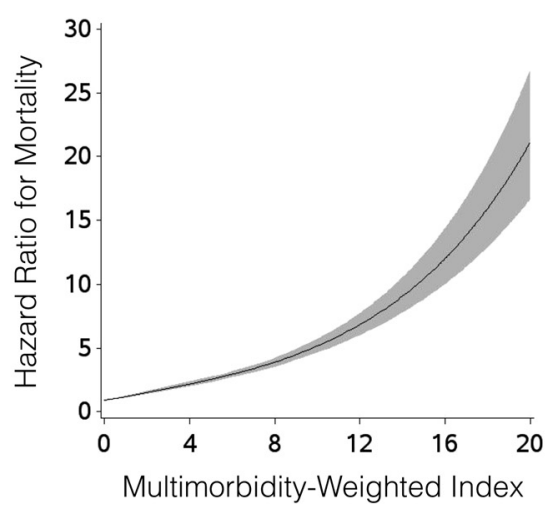

C HPFS 1992-2016 mortality by suicide

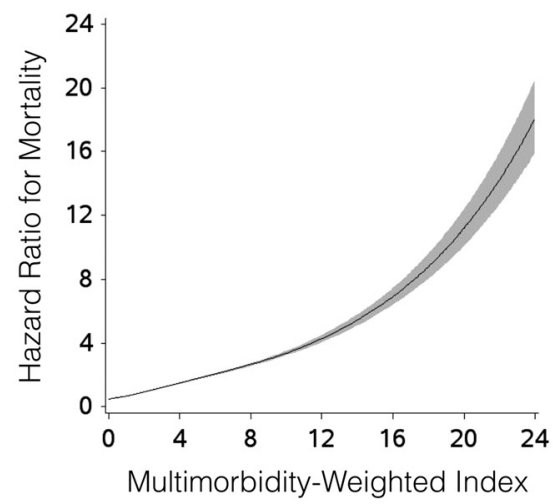

Figure 1. Restricted cubic splines for suicide mortality by multimorbidity-weighted index (MWI) in (A) the Nurses' Health Study (NHS), (B), the Nurses' Health Study II, and (C) the Health Professionals Follow-up Study, from 1992 or 1993 to 2016. Curves represent the hazard ratio (HR) for suicide mortality (solid line) with corresponding 95\% confidence intervals (gray surrounding lines and cloud). At lower values of the MWI, there were essentially linear relationships with suicide mortality; at higher values of the MWI, there were disproportionately higher HRs for suicide mortality.

observed with MWI as a continuous measure (Supplemental Table S4).

Among younger women in NHS II, who tended to have less multimorbidity, the association between MWI on MCS was greater than for women in NHS. In unadjusted models, women with the highest quartile MWI had a $-1.25(95 \%$ $\mathrm{CI}=-1.44$ to -1.06 ) worse MCS compared with those in the lowest quartile MWI. Each point increase in MWI was associated with a 0.26 -point decrease in MCS (Supplemental Table S4 and Figure 3). In models adjusting for covariates except baseline MCS, the effect of each point increase in MCS was greater. Further adjustment for baseline MCS attenuated the association modestly. For comparison, the association of 1-point increase in MWI with MCS was 5 to 10 times greater than that of a 1-year increase in age.

Among individual mental HRQOL scales, MWI was associated with the greatest decrement in social functioning, followed by general health, vitality, role-emotional, and mental health. However, we observed statistically significant adverse associations of MWI with lower mental HRQOL for all scales.

\begin{tabular}{|c|c|c|c|c|c|c|c|c|}
\hline \multirow{2}{*}{$\frac{\text { No. suicide deaths }}{\text { Model }}$} & \multicolumn{2}{|c|}{$\frac{\text { NHS (N = } 102854)}{77}$} & \multicolumn{2}{|c|}{$\frac{\text { NHS II (N = } 103584)}{110}$} & \multicolumn{2}{|c|}{$\frac{\text { HPFS }(\mathrm{N}=45564)}{154}$} & \multicolumn{2}{|c|}{$\begin{array}{c}\text { Pooled cohorts }(\mathrm{N}=252003) \\
341\end{array}$} \\
\hline & HR (95\% CI) & $P$-value & HR (95\% Cl) & $P$-value & HR (95\% Cl) & $P$-value & HR (95\% Cl) & $P$-value \\
\hline \multicolumn{9}{|l|}{ MWI, continuous } \\
\hline Model $2^{\mathrm{C}}$ & 1.09 (1.03-1.14) & .002 & 1.13 (1.06-1.19) & $<.001$ & $1.05(1.00-1.11)$ & .060 & 1.09 (1.06-1.12) & $<.001$ \\
\hline \multicolumn{9}{|l|}{ MWI, unadjusted } \\
\hline Q4 vs Q1 & 2.98 (1.54-5.80) & .001 & $3.95(2.41-6.48)$ & $<.001$ & 2.36 (1.51-3.69) & $<.001$ & 3.06 (2.76-3.35) & $<.001$ \\
\hline Q3 vs Q1 & $1.14(0.53-2.47)$ & .739 & $1.87(1.09-3.20)$ & .023 & $2.03(1.32-3.14)$ & .001 & $1.84(1.53-2.14)$ & $<.001$ \\
\hline Q2 vs Q1 & $1.71(0.83-3.53)$ & .145 & 1.82 (1.03-3.20) & .038 & $1.07(0.63-1.81)$ & .811 & $1.48(1.14-1.82)$ & $<.001$ \\
\hline Q1 ${ }^{d}$ (reference) & 1.0 & & 1.0 & & 1.0 & & 1.0 & \\
\hline \multicolumn{9}{|l|}{$\mathrm{MWI}$, model $2^{\mathrm{C}}$} \\
\hline Q4 vs Q1 & $3.01(1.48-6.11)$ & .002 & 3.04 (1.82-5.09) & $<.001$ & $1.74(1.08-2.81)$ & .022 & $2.47(2.16-2.79)$ & $<.001$ \\
\hline Q3 vs Q1 & $1.23(0.56-2.69)$ & .612 & $1.60(0.93-2.77)$ & .090 & $1.76(1.13-2.74)$ & .012 & $1.62(1.30-1.94)$ & $<.001$ \\
\hline Q2 vs Q1 & 1.78 (0.86-3.69) & .121 & $1.70(0.97-3.00)$ & .065 & $1.01(0.59-1.71)$ & .985 & 1.43 (1.09-1.77) & $<.001$ \\
\hline $\mathrm{Q1}^{\mathrm{d}}$ (reference) & 1.0 & & 1.0 & & 1.0 & & 1.0 & \\
\hline
\end{tabular}

${ }^{a}$ Continuous and quartiles between 1992 (NHS, HPFS) or 1993 (NHS II) to 2016.

${ }^{\mathrm{b}}$ Adjusted for age, race, body mass index, smoking status, geographic region.

${ }^{c}$ Further adjusted for alcohol consumption ( $0 \mathrm{~g} / \mathrm{d}$ or tertile of intake) and coffee intake, marital status, living alone, and additionally in the NHS, religious service attendance.

${ }^{\mathrm{d}}$ For NHS II, the referent category Q1 is MWI values of 0 , due to the left skew in this cohort with many participants with MWI values of 0 (ie, ties to 0 ). $\mathrm{CI}=$ confidence interval; HPFS = Health Professionals Follow-up Study; HR = hazard ratio; MWI = multimorbidity-weighted index; NA = not available; NHS = Nurses' Health Study; NHS II = Nurses' Health Study II; Q = quartile. 
A

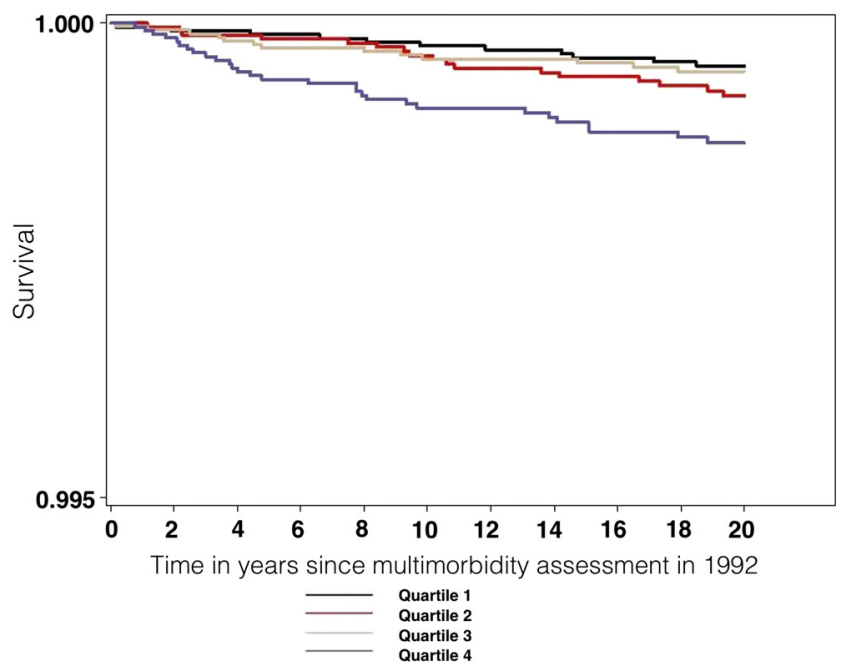

B

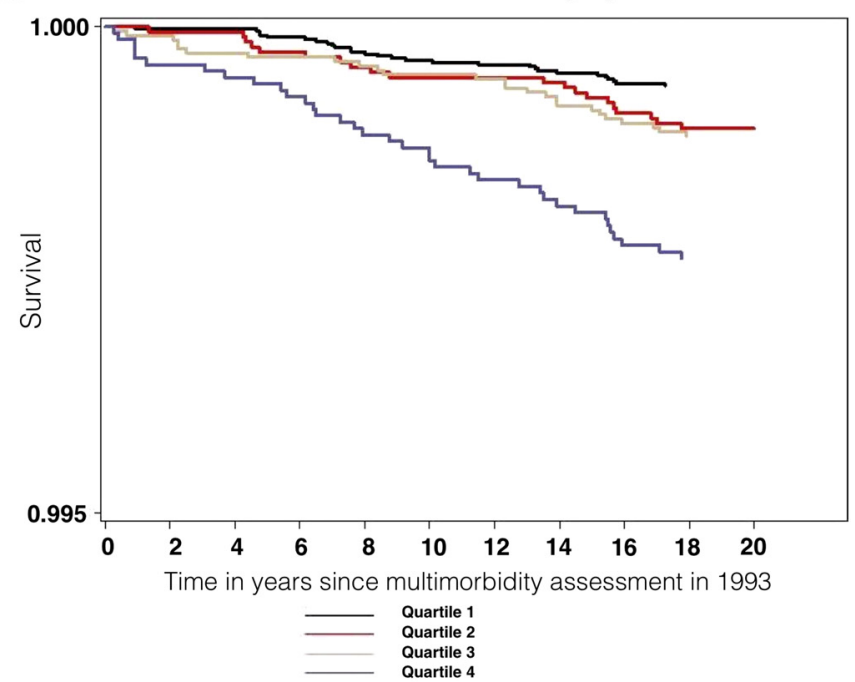

C HPFS 1992-2016 mortality by suicide

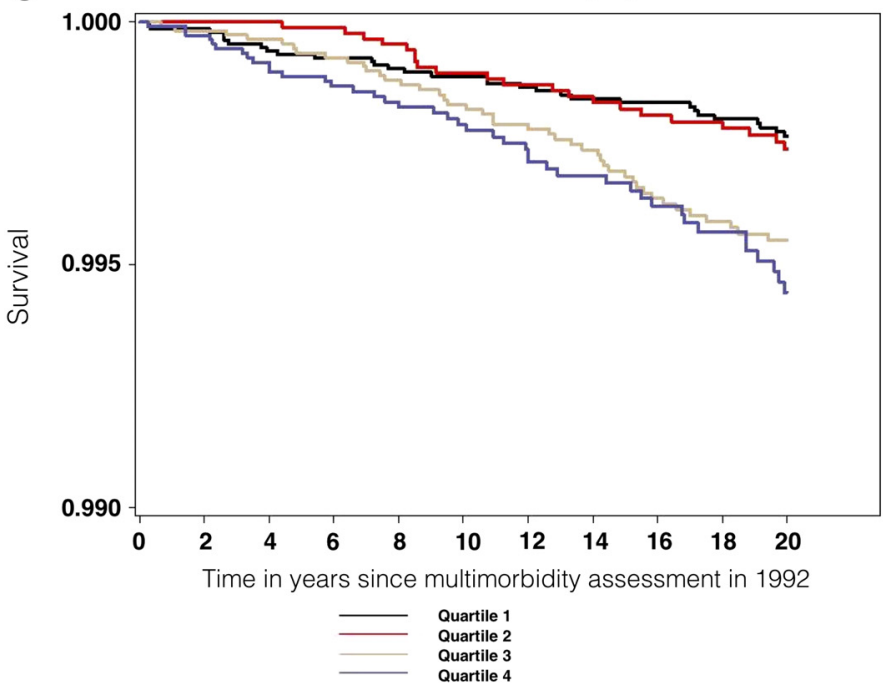

Figure 2. Kaplan-Meier survival curves for suicide mortality by multimorbidity-weighted index (MWI) quartiles in (A), the Nurses' Health Study (NHS), (B), the Nurses' Health Study II, and (C), the Health Professionals Follow-up Study, from 1992 or 1993 to 2016. There was suggestion of a dose-response association between increasing quartiles of the MWI and worse survival over 24 years of follow-up for suicide mortality. All nonsuicide causes of death and nondeaths were censored.

For comparison with mental HRQOL, we show associations between multimorbidity and physical HRQOL (Supplemental Table S5 and Figure 3). In general, and as expected from its weighting to current physical functioning, MWI was associated with decrements in future physical HRQOL larger than for mental HRQOL, with consistent associations across subscales. As with mental HRQOL, each point increase in MWI was associated with worse physical HRQOL on average in younger women (NHS II) than older women (NHS), although younger women were less likely to have multimorbidity.

\section{DISCUSSION}

In this large prospective study of 252002 young through older community-dwelling adults, multimorbidity was strongly associated with increased suicide mortality and decreased mental HRQOL. Those with the highest quartile of multimorbidity had a two - fourfold higher risk of suicide mortality compared with those in the lowest quartile. Collectively, these findings suggest multimorbidity has an important role in the progression of HRQOL across all mental and physical domains and with risk of suicide mortality, spanning the full continuum of multimorbidity.

Our finding that multimorbidity is associated with increased suicide is consistent with and extends previous studies. Prior studies reported physical health conditions, even after adjustment for mental health and substance abuse diagnoses, were associated with increased suicide behavior and mortality. ${ }^{7,9}$ Webb et al similarly reported a doseresponse association with more physical conditions and risk of self-harm, independent of depression in women only. ${ }^{34}$ Another study reported multimorbidity from mental and physical health conditions was associated with increased suicidal ideation, but not beyond that of having a common mental disorder only. ${ }^{35}$ Although mental health conditions 

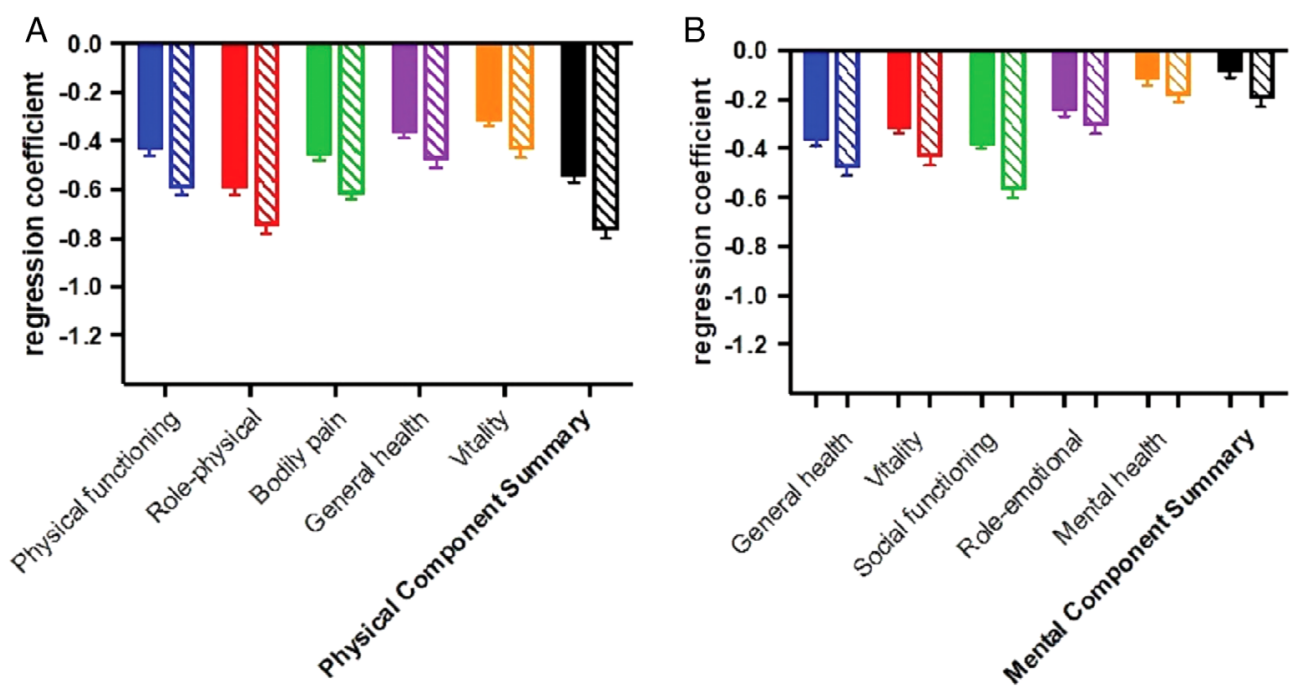

Figure 3. Short Form-36 scales and summary component measures per point increase in the multimorbidity-weighted index (MWI) after 8-year follow-up in the Nurses' Health Study (left-hand solid-colored columns, 1992-2000) and Nurses' Health Study II (right-hand diagonal hashed columns, 1993-2001) in adjusted models. A, Physical health-related quality of life scales and the physical component summary measure. B, Mental health-related quality of life scales and the mental component summary measure. Histograms represent the regression coefficients with corresponding $95 \%$ confidence intervals as the error bars. Each point increase in the MWI was associated with significantly worse health-related quality of life across all mental and physical scales and summary component measures. [Color figure can be viewed at wileyonlinelibrary.com]

predicted suicide ideation, it was less helpful in determining who would make an attempt. In contrast to studies of suicide behavior, we examined completed suicide. We also had a large sample size spanning young through older adults with a long duration of follow-up, comprehensive assessment of multimorbidity accounting for disease severity, and broad control for confounding over several domains. Further, by examining health professionals, we expect less misclassification of self-reported chronic conditions and thus findings that more closely mimic relationships expected using diseases identified in medical records.

Previous studies also reported on the association between multimorbidity and decreased HRQOL using various measures but were largely cross-sectional designs at midlife ${ }^{36,37}$ and in older adults ${ }^{38}$ without adjustment for current HRQOL. Our prospective study demonstrated that the burden of multimorbidity on HRQOL persisted 8 years later. We adjusted for current HRQOL to avoid ambiguities of reverse causality and to estimate the effect of incident multimorbidity. Although MWI was developed using concurrent physical functioning, MWI was also associated with mental HRQOL, although that SF-36 mental and PCS measures are orthogonal (uncorrelated) by design. Indeed, while MWI predicted PCS years later with nearly the same predicted weighting as at baseline, MWI was associated with a decline in MCS roughly one quarter that of PCS, confirming multimorbidity has a clear, quantifiable link to future mental health even when indexed to physical health.

Although younger women are less likely to have multimorbidity than older women, those who did reported poor HRQOL worse than that of older women. There are several possible explanations for this finding. First, some conditions affecting younger women may be more virulent, particularly early in the disease course. For example, connective tissue disease, inflammatory bowel disease, and breast cancer had a greater impact on physical functioning in younger NHS II women than older NHS women. ${ }^{14}$ Second, younger adults may have been more recently diagnosed and/or had less time for treatment and adaptation than older adults. For example, stroke is often worse earlier in the disease course. Finally, HRQOL is a self-reported perception of the impact of disease on one's state of health. A "response shift" may occur whereby there is a change in the participant's internal standards or values, resulting in a recalibration of the SF-36 or reconceptualization of HRQOL over time as other aspects in life change. ${ }^{39}$ Social comparisons may also contribute to response shift. For example, older adults may downplay their limitations if they compare themselves with peers with worse disability. In contrast, younger adults may compare themselves with healthy peers.

Our study has potential limitations. First, MWI includes mostly physical conditions and is thus an underestimate of mental conditions. The original MWI included self-reported conditions, but for robustness, we added to MWI morbidities from antidepressant and tranquilizer use. Opiate use was unavailable but much less commonly used at baseline. ${ }^{40}$ Nevertheless, MWI remained associated with mental HRQOL, consistent with studies reporting physical conditions, irrespective of comorbid mental conditions, are associated with increased suicide behavior and mortality. ${ }^{7,9}$ Overall, these features highlight the role of physical multimorbidity in predicting future mental health but suggest that indices incorporating mental health conditions would have even stronger effects.

Another limitation was participants were predominantly white health professionals, although geographically diverse with disease prevalences and lifestyles reflecting those of general US and other industrialized populations. ${ }^{41-44}$ Health professionals may have easier access to medications compared with the general population, ${ }^{45,46}$ although we were 
unable to compare suicide methods by occupation. Further studies on suicide mortality are needed in diverse populations. Finally, long-term HRQOL results are restricted to women. One scale, physical functioning, was available in men and persisted over follow-up. ${ }^{21}$

In this prospective study, an easily measured multimorbidity index capturing a comprehensive inventory of conditions was strongly associated with increased suicide mortality and decreased mental HRQOL in young through older community-dwelling adults. These results support a role for MWI in capturing mental health, despite being weighted specifically to physical functioning. MWI may serve as a useful tool and Web-based or electronic medical record portal application for healthcare providers considering broader, long-term consequences of multimorbidity.

\section{ACKNOWLEDGMENTS}

We thank participants of the Nurses' Health Study, Nurses' Health Study II, and Health Professionals Follow-up Study. These cohorts are based at the Channing Division of Network Medicine, Department of Medicine, Brigham and Women's Hospital, Boston, Massachusetts.

Conflict of Interest: The authors have declared no conflict of interest for this article.

Author Contributions: Melissa Y. Wei and Kenneth J. Mukamal conceived and designed the work, analyzed and interpreted the data for the study, and critically revised the manuscript for intellectual content. Melissa Y. Wei acquired the data, performed the analysis, and drafted the manuscript.

Sponsor's Role: None.

\section{REFERENCES}

1. Centers for Disease Control and Prevention (CDC). Deaths and Mortality. Atlanta, GA: CDC, 2017.

2. Hedegaard H, Curtin S, Warner M. Suicide Rates in the United States Continue to Increase. Hyattsville, MD: US Department of Health and Human Services, 2018.

3. Ahmedani BK, Simon GE, Stewart C et al. Health care contacts in the year before suicide death. J Gen Intern Med. 2014;29:870-877.

4. Cavanagh JT, Carson AJ, Sharpe M et al. Psychological autopsy studies of suicide: a systematic review. Psychol Med 2003;33:395-405.

5. Bohnert KM, Ilgen MA, Louzon $S$ et al. Substance use disorders and the risk of suicide mortality among men and women in the US Veterans Health Administration. Addiction 2017;112:1193-1201.

6. Ohrnberger J, Fichera E, Sutton M. The dynamics of physical and mental health in the older population. J Econ Ageing 2017;9:52-62.

7. MacLean J, Kinley DJ, Jacobi F et al. The relationship between physical conditions and suicidal behavior among those with mood disorders. J Affect Disord 2011;130:245-250.

8. Scott KM, Hwang I, Chiu WT et al. Chronic physical conditions and their association with first onset of suicidal behavior in the world mental health surveys. Psychosom Med 2010;72:712-719.

9. Ahmedani BK, Peterson EL, Hu Y et al. Major physical health conditions and risk of suicide. Am J Prev Med 2017;53:308-315.

10. Juurlink DN, Herrmann N, Szalai JP et al. Medical illness and the risk of suicide in the elderly. Arch Intern Med 2004;164:1179-1184.

11. Waern M, Rubenowitz E, Runeson B et al. Burden of illness and suicide in elderly people: case-control study. BMJ 2002;324:1355.

12. Wei MY, Kabeto MU, Galecki AT et al. Physical functioning decline and mortality in older adults with multimorbidity: joint modeling of longitudinal and survival data. J Gerontol A Biol Sci Med Sci 2018. https://doi.org/10. 1093/gerona/gly038.

13. Goodman RA, Ling SM, Briss PA et al. Multimorbidity patterns in the United States: implications for research and clinical practice. J Gerontol A Biol Sci Med Sci 2016;71:215-220.
14. Wei MY, Kawachi I, Okereke OI et al. Diverse cumulative impact of chronic diseases on physical health-related quality of life: implications for a measure of multimorbidity. Am J Epidemiol 2016;184:357-365.

15. Wei MY, Kabeto MU, Langa KM et al. Multimorbidity and physical and cognitive function in nationally-representative US adults: performance of a new multimorbidity-weighted index. J Gerontol A Biol Sci Med Sci 2018;73: 225-232.

16. Ware JE, Jr. SF-36 Health Survey Manual and Interpretation Guide. Boston, MA: The Health Institute, New England Medical Center Hospitals, Inc., 1993.

17. Otchet F, Carey MS, Adam L. General health and psychological symptom status in pregnancy and the puerperium: what is normal? Obstet Gynecol 1999;94:935-941.

18. Rich-Edwards JW, Corsano KA, Stampfer MJ. Test of the National Death Index and Equifax Nationwide Death Search. Am J Epidemiol 1994;140: 1016-1019.

19. Stampfer MJ, Willett WC, Speizer FE et al. Test of the National Death Index. Am J Epidemiol 1984;119:837-839.

20. Brazier JE, Harper R, Jones NM et al. Validating the SF-36 health survey questionnaire: new outcome measure for primary care. BMJ 1992;305: 160-164.

21. Wei MY, Mukamal KJ. Multimorbidity, physical functioning, and long-term mortality in three prospective cohorts of community-dwelling adults. Am J Epidemiol 2018;187:103-112.

22. Staimez LR, Wei MY, Kim M et al. Multimorbidity of four cardiometabolic and chronic pulmonary disease groups: prevalence and attributable fraction in US adults, 2007-2012. J Comorbidity 2017;7:22-32.

23. Mukamal KJ, Rimm EB, Kawachi I et al. Body mass index and risk of suicide among one million US adults. Epidemiology 2010;21:82-86.

24. Schrieks IC, Wei MY, Rimm EB et al. Bidirectional associations between alcohol consumption and health-related quality of life amongst young and middle-aged women. J Intern Med 2016;279:376-387.

25. Ding M, Satija A, Bhupathiraju SN et al. Association of coffee consumption with total and cause-specific mortality in 3 large prospective cohorts. Circulation 2015;132:2305-2315.

26. Olaya B, Domenech-Abella J, Moneta MV et al. All-cause mortality and multimorbidity in older adults: The role of social support and loneliness. Exp Gerontol 2017;99:120-126.

27. VanderWeele TJ, Li S, Tsai AC et al. Association between religious service attendance and lower suicide rates among US women. JAMA Psychiatry 2016;73:845-851.

28. So Y, Lin G, Johnston G; SAS Institute, Inc. Using the PHREG procedure to analyze competing-risks data. https://support.sas.com/rnd/app/stat/papers/2014/ competingrisk2014.pdf. Accessed October 31, 2018.

29. Efron B. The efficiency of Cox's likelihood function for censored data. J Am Stat Assoc 1977;72:557-565.

30. Hertzmark E, Spiegelman D. The SAS METAANAL Macro. https://cdn1.sph. harvard.edu/wp-content/uploads/sites/271/2012/09/metaanal_user_manual_-_524-2012.pdf. Accessed October 31, 2018.

31. Durrleman S, Simon R. Flexible regression models with cubic splines. Stat Med 1989;8:551-561.

32. Hastings C, Mosteller F, Tukey JW et al. Low moments for small samples: a comparative study of order statistics. Ann Math Stat. 1947;18:413-426.

33. Vickers AJ, Altman DG. Statistics notes: analysing controlled trials with baseline and follow up measurements. BMJ 2001;323:1123-1124.

34. Webb RT, Kontopantelis E, Doran T et al. Risk of self-harm in physically ill patients in UK primary care. J Psychosom Res 2012;73:92-97.

35. Kavalidou K, Smith DJ, O'Connor RC. The role of physical and mental health multimorbidity in suicidal ideation. J Affect Disord 2017;209: $80-85$.

36. Kanesarajaha J, Waller M, Whitty JA et al. Multimorbidity and quality of life at mid-life: A systematic review of general population studies. Maturitas 2018;109:53-62.

37. Wang L, Palmer AJ, Cocker F et al. Multimorbidity and health-related quality of life (HRQoL) in a nationally representative population sample: implications of count versus cluster method for defining multimorbidity on HRQoL. Health Qual Life Outcomes 2017;15:7.

38. Hunger M, Thorand B, Schunk $\mathrm{M}$ et al. Multimorbidity and health-related quality of life in the older population: results from the German KORA-age study. Health Qual Life Outcomes 2011;9:53.

39. Ubel PA, Loewenstein G, Jepson C. Whose quality of life? A commentary exploring discrepancies between health state evaluations of patients and the general public. Qual Life Res 2003;12:599-607.

40. Compton WM, Conway KP, Stinson FS et al. Changes in the prevalence of major depression and comorbid substance use disorders in the United States between 1991-1992 and 2001-2002. Am J Psychiatry 2006;163: 2141-2147. 
41. Lochner KA, Cox CS. Prevalence of multiple chronic conditions among Medicare beneficiaries, United States, 2010. Prev Chronic Dis 2013;10:E61.

42. van Baal PH, Engelfriet PM, Boshuizen HC et al. Co-occurrence of diabetes, myocardial infarction, stroke, and cancer: quantifying age patterns in the Dutch population using health survey data. Popul Health Metr 2011;9:51.

43. Schafer I, von Leitner EC, Schon G et al. Multimorbidity patterns in the elderly: a new approach of disease clustering identifies complex interrelations between chronic conditions. PLoS One 2010;5:e15941.

44. Schnell K, Weiss CO, Lee $\mathrm{T}$ et al. The prevalence of clinically-relevant comorbid conditions in patients with physician-diagnosed COPD: A cross-sectional study using data from NHANES 1999-2008. BMC Pulm Med 2012;12:26.

45. Milner AJ, Maheen H, Bismark MM et al. Suicide by health professionals: A retrospective mortality study in Australia, 2001-2012. Med J Aust 2016;205: 260-265.

46. Hawton K, Agerbo E, Simkin S et al. Risk of suicide in medical and related occupational groups: A national study based on Danish case populationbased registers. J Affect Disord 2011;134:320-326.

\section{SUPPORTING INFORMATION}

Additional Supporting Information may be found in the online version of this article.

Supplementary Table S1. Short Form-36 scales and summary component measures and correlations between baseline and follow-up in the Nurses' Health Study (NHS) (1992-2000) and NHS II (1993-2001).

Supplemental Table S2. Hazard ratios for suicide mortality by multimorbidity-weighted index (continuous and quartiles) that includes morbidities from medications (antidepressants, and minor and major tranquilizers ${ }^{\mathrm{a}}$ ) between 1992 (Health Professionals Follow-up Study) or 1993 (Nurses' Health Study II) to 2016.

Supplemental Table S3. Hazard ratios for suicide mortality by multimorbidity-weighted index (continuous and quartiles) for individuals without any mental or substance use conditions, between 1992 (Health Professionals Follow-up Study) or 1993 (Nurses' Health Study II) to 2016.

Supplementary Table S4. Long-term Short Form-36 physical and mental health-related quality of life scales and summary measures per point increase in the multimorbidity-weighted index in the Nurses' Health Study (1992-2000) and NHS II (1993-2001).

Supplemental Table S5. Long-term Short Form-36 physical and mental health-related quality of life scales and summary measures by quartiles of the multimorbidity-weighted index in the Nurses' Health Study (1992-2000) and NHS II (1993-2001). 